\title{
The impact of telematics on traffic safety
}

\author{
DOI: https://doi.org/10.7307/ptsm.2020.10
}

\author{
Igor Jelić ${ }^{1}$, Maja Balenović ${ }^{2}$ \\ School for road traffic, Zagreb ${ }^{1}$; School for road traffic, Zagreb ${ }^{2}$
}

\begin{tabular}{l} 
Keywords \\
\hline Telematics \\
Safety \\
Traffic \\
Roads
\end{tabular}

\section{EU road safety}

The number of people killed in road crashes around the world continues to increase. According to the World Health Organisation's "Global Status Report on Road Safety", it reached 1.35 million in 2016 alone. This means that, worldwide, more people die as a result of road traffic injuries than from HIV/AIDS, tuberculosis or diarrhoeal diseases. And road crashes are now the most common cause of death for children and young people between 5 and 29 worldwide. Compared to the global situation, Europe is doing relatively well, thanks to determined action at EU, national, regional and local level. Between 2001 and 2010, the number of road deaths in the EU decreased by 43\%, and between 2010 and 2018 by another $21 \%$. However, 25,100 people still lost their lives on EU roads in 2018 and about 135,000 were seriously injured. This is an unacceptable and unnecessary human and social price to pay for mobility. In monetary terms alone, the yearly cost of road crashes in the EU has been estimated in a new study to be around EUR 280 billion, equivalent to about $2 \%$ of GDP5.[1]

Progress in reducing EU-wide road fatality rates has stagnated in recent years. It appears highly unlikely that the EU's current medium term target, to halve the number of road deaths between 2010 and 20206, will be reached. Even less progress has been made in preventing serious injuries[1]. In some well-performing countries, fatality figures have even risen again in recent years. Whilst certain fluctuations are to be expected especially if the absolute figures and sample sizes are small, these cases need further analysis at national and EU level, along with analysis of shifts in the accident types and user groups involved, in order to allow a timely and effective policy response[1].

The EU has reaffirmed its ambitious long-term goal, to move close to zero deaths by 2050 ("Vision Zero"). To move towards these goals, a new approach is set out in the "Europe on the Move" Communication. First of all, the mindset of "Vision Zero" needs to take hold more than it has so far, both among policy makers and in society at large[1]. Road crashes are "silent killers", in that they often go virtually unnoticed in the public sphere, even though, taken together, they kill as many people - around 500 - as fit into a jumbo jet every 
week, in Europe alone. We do not accept deaths in the air, and we should no longer accept them on the road the premise that no loss of life is acceptable needs to inform all decision making on road safety.

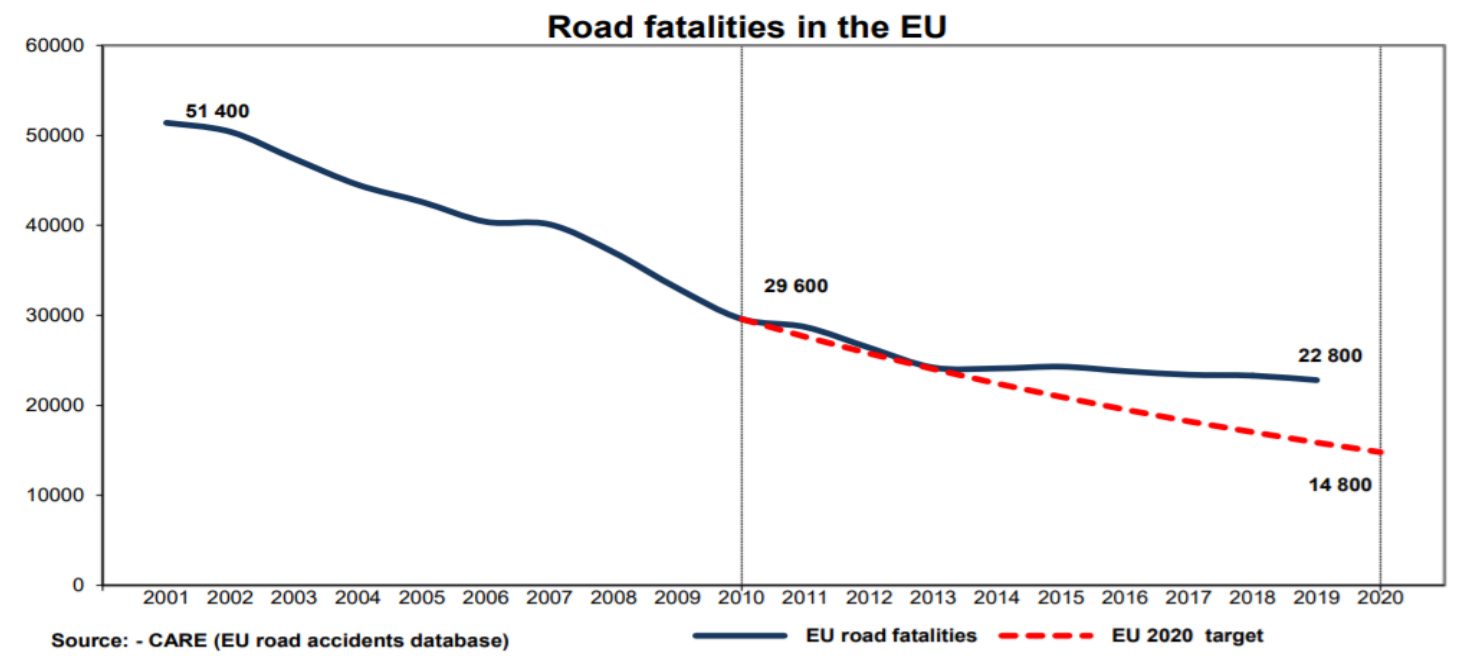

Figure 1. Evolution of EU road fatalities and targets for 2001-2020

Source: [1]

\section{Telematics solution in vehicles}

Telematics is a term that has been created to describe technologies and applications which make integrated use of telecommunications and informatics. More specifically, telematics is the discipline concerned with the transfer of data and services to mobile locations such as vehicles, containers, or people. The underlying technologies are often referred to as Information and Communications Technologies or ICT. Telematics has its origins in military localisation applications, but the term is now mostly used as a synonym for vehicle telematics (also known as Road Transport and Traffic Telematics or RTTT in Europe, and Intelligent Transport Systems or ITS in the United Kingdom and United States). Vehicle telematics applications are delivered through in-vehicle devices that as core technologies typically contain satellite localisation such as Global Positioning System (GPS) and cellular communications such as General Packet Radio Service (GPRS).

Innovations in vehicle technology can help both mitigate the severity of crashes and reduce the likelihood of crashes through on one hand passive safety features such as safety belts, airbags, and general crashworthiness of vehicles that protect occupants when a collision is inevitable, and on the other hand active safety features, such as Advanced Emergency Braking, Intelligent Speed Assistance, Stability Control and Lane Departure Warning that may prevent accidents from happening altogether.

\section{eCall}

eCall is a system that provides an automated message to the emergency services following a road crash which includes the precise crash location. The in-vehicle eCall is an emergency call (an E112 wireless call) generated either manually by the vehicle occupants by pushing a button or automatically via activation of in-vehicle sensors after a crash. When activated, the in-vehicle eCall device will establish an emergency call carrying both voice and data directly to the nearest emergency services (normally the nearest 112 Public Safety Answering Point, PSAP). The voice call enables vehicle occupants to communicate with the trained eCall operator. At the same time, a minimum set of data will be sent to the eCall operator receiving the voice call. The minimum set of data contains information about the incident including time, precise location, vehicle identification, eCall status (as a minimum, indication if eCall has been manually or automatically triggered) and information about a possible service provider.

A prospective Finnish study has estimated that such a system might reduce between 4-8\% of road deaths and $5-10 \%$ of motor vehicle occupant deaths in Finland [2]. The study assumed that all vehicles were equipped 
with the eCall terminal and that each terminal would function properly. The study was unable to evaluate the impact of the precise location information given by eCall on the swifter arrival of rescue units at the accident site in the evaluation of decrease in road traffic deaths. The overall impact of the system, which involves additional players, has not been evaluated.

The Finnish study noted that through "the comparison of the 4-8\% decrease in traffic accident fatalities arrived at in this study with the figures of other European studies one can see that the results are similar to the German (5\%) and Dutch (7\%) estimations. The estimations in Sweden (2-4\%) and Great Britain (2\%) are smaller and the estimate for the whole 25 member state EU area (5-15\%) greater than the estimate in this study. The American estimation for the decrease in traffic accident fatalities based on field studies was smaller (2-3\%) than in this study. The estimate made by the doctors was, however, greater (9-11\%) [2].

\section{Increase efficiency, job satisfaction and reduce operating costs}

Real-time communication coupled with routing apps can divert drivers away from road delays and toward high-priority loads. Adding even a few minutes of driving time to each driver's daily hours of service (HOS) can boost overall fleet utilization.

With electronic logs, drivers have more control over their daily routines, which is one of the top contributors to job satisfaction. The drivers know how much time they have left to work and can better collaborate with dispatchers to meet customer demands. With smartphones and tablets with them at all times, they can engage in training at a time and place that is convenient for them.

Saving even a few minutes of idling time across a fleet leads to huge fuel savings. Better fleet and driver utilization can lift equipment availability to meet customer demand with existing assets. Connecting with drivers via cab-mounted tablets, fleet managers can instantaneously update drivers on detours, reroute them around heavy traffic and direct them to preferred fuel vendors.

\section{Smart road technology}

The road is an often-overlooked part of the modern transport infrastructure. We've all heard of self-driving cars, navigation apps, and ride-hailing services. But as it turns out, the road itself can be a platform for an amazing array of innovations. Roads can be upgraded with communication, lighting and power transmission technologies that can support sustainability, improve safety, and transform the driving experience. It is estimated that road infrastructure and road surroundings are a contributing factor in more than $30 \%$ of crashes. In the last few years, technology has changed almost everything around us. For instance, it has completely changed the way we used to spend money, communicate or even drive. Certain technologies like telematics have revolutionized both motor insurance and road safety [3].

Smart roads use Internet of Things (IoT) devices to make driving safer, more efficient, and greener. Smart roads combine physical infrastructures such as sensors and solar panels with software infrastructure like AI and big data. Smart road technologies are embedded in roads and can improve visibility, generate energy, communicate with autonomous vehicles, monitor road conditions, and more.

Smart road technologies [3]:

1. Solar powered roadways - Photovoltaic cells are embedded within hexagonal panels made of tempered glass, which are used to pave roads. These panels contain LEDs, microprocessors, snow-melting heating devices and inductive charging capability for electric vehicles when driving. Glass is renewable and can be engineered to be stronger than steel, and to allow cars to stop safely even when traveling at high speeds. While this idea has gained widespread support, attracting over $\$ 2$ million in crowdfunding, scalability is a challenge as it remains expensive. 
2. Smart pavement - Specially engineered roadways fitted with smart features, including sensors that monitor and report changing road conditions, and WiFi transmitters that provide broadband services to vehicles, homes and businesses. The smart pavement can also charge electric cars as they drive.

3. Glow in the dark roads - Glowing markers painted onto existing roadway surfaces use a photo-luminescent powder that absorbs and stores daylight. The 500m long strips glow for 8 hours after dark. This technology is still in the testing phase, and the glow is not yet consistent, but it could be more cost-effective than traditional road lighting.

4. Interactive lights - Road lights activated by motion sensors to illuminate a particular section of the road as a car approaches. The lights dim once the car passes. Suited for roads with less traffic, interactive lights provide night visibility as needed and reduce energy wastage when there are no cars. One design, developed by the Dutch Studio Roosegaarde, uses wind generated by passing vehicles to power lights.

5. Electric priority lane for charging electric vehicles - Embedded cables generate magnetic fields that charge electric vehicles while driving. A receiver coil in the vehicle picks up electromagnetic oscillations from a transmitter coil embedded in the road and converts them to AC, which can then power the car. Inductive charging technology already exists for static cars, but future wireless technology could charge batteries while in motion.

6. Weather detection - Networks of AI-integrated sensors detect weather conditions that impact road safety. Road Weather Information Systems (RWIS) in use today are limited because they only collect data from a small set of weather stations. A larger future network could use automated weather stations to collect atmospheric and weather data and instantly upload it to the cloud. Dynamic temperature-sensitive paint could be used to highlight invisible roadway conditions like black ice.

7. Traffic detection - Data that helps travelers plan their routes. Sensors lining highways monitor traffic flow and weight load, warn drivers of traffic jams, and automatically alert the authorities about accidents. Fiberoptic cables embedded in the road detect wear and tear, and communication between vehicles and roads can improve traffic management. For example, Rapid Flow Technologies uses artificial intelligence (AI) to manage traffic lights, which respond to each other and to cars.

Many governments and transport authorities understand the value of smart road technologies. However, developing smart city infrastructure at scale can be costly and complex. Leaders can break down smart road projects into chunks, starting with low-investment, narrow-scale initiatives that can provide initial value, setting the stage for high-investment, large-scale efforts. In the early days of motor-powered mobility, cars were available, but there was no suitable road infrastructure; the first private cars were hardly more effective than horse-driven wagons. Gradually, authorities recognized that only a major investment in road infrastructure would help the population reap the benefits of new transport technology. Similarly, today's municipal governments and inter-city transport authorities should wake up to the importance of smart roads, as an essential platform for mobility innovation. Smart roads will power smarter cars, empower drivers, and provide governments with unprecedented visibility and control over the living fabric of motor-based traffic. [3]

\section{The key of attributes of smart roads}

We must distinguish the attributes with a direct influence on road design, construction and maintenance - we will name them structural aspects - from those aspects related to the subjective perceptions of users driving through our roads, to then reconcile those structural aspects with the users' expectations.

\section{Structural acttributes}

Smart Roads are associated with structural aspects such as, although not limited to, the following [4]: 
- Optimal environmental integration and energy efficiency: This refers to the capacity of our road systems to respect high environmental protection levels, minimising the associated environmental impacts whilst also allowing all users to enjoy the surrounding region through careful landscape integration. At the same time, this approach involves developing optimised models for the lowest possible energy consumption and emission levels, maximizing the use of recycled and waste materials. To this end, it is important to take into account the entire life cycle of a road infrastructure.

- Optimal service quality: Through advanced traffic management it is possible to optimise the use of existing road networks, limiting congestion during hours of peak demand, and at the same time distributing traffic as evenly as possible to maximise the service levels offered at all times. This approach relies on the streamlined transmission of real-time information to road users and on fostering the interaction between users and the information presented to them.

- Economic sustainability: Highways designed for the mass transport of people and goods are economically justified in most of the cases. This principle is all the more important when it comes to evaluating the cost of operating and maintaining this type of infrastructures. It is therefore highly advised to proceed with detailed cost-benefit analyses with a view to justifying the usefulness of every road project. Full debate must also be undertaken when seeking public-private partnerships financing the construction of new infrastructures and the maintenance and upgrading of existing networks.

- Improved safety: This means following internationally-recognised best practices in the design of new road infrastructures and in the implementation of the best possible safety solutions - for all users - in the maintenance, upgrading and modernisation of existing infrastructures. Collaborative efforts in this regard should contribute to reducing road mortality and injury rates through more effective design, construction and maintenance of road infrastructures.

- Coverage of externalities: The external costs arising from the movement of people and goods on roads must be kept to a minimum through the development of technologies to absorb emissions and noise, minimise the probability of and mitigate the impacts of accidents, and in addition provide maximum travel time in free-flowing traffic.

- Assurance of regional cohesion: Conventional roads are a paradigm for effective regional development, as they offer all users an ideal door-to-door transport alternative providing access to all social services (e.g., leisure, culture, health, education and other infrastructures). At the same time, it is necessary not to overlook the importance of roads in urban and suburban areas, which are fundamental for those living both in and outside cities.

- Focus on co-modality: It is also important to acknowledge the key role roads play in a realistic scenario for the effective use of different transport modes in combination, with a view to realising a sustainable utilization of transportation resources. Roads are inevitably an essential link in the modal chain as they are the sole mode providing access to ports, airports, commuter stations, bus connection points and so on. This unique attribute of road infrastructures consequently needs to be highlighted as a complementary factor in relation to other transport modes.

- Adaptability of services offered: Over time, all transport modes have successfully adapted their offer to the expectations of the various types of users (commuter and high-speed trains, rail transport of goods, light rail and underground transit, international flights, private jets, mid-haul flights, etc.) and, in so doing, the related infrastructures have been adapted to each type of mobility demand. On the other hand, roads have provided a solution to all users and for all types of travel around the clock, 365 days a year, based on two main offerings: conventional roads and divided highways. The time has come for road infrastructures also to modify their type and characteristics through an optimised design and differentiation amongst uses and users per itinerary (e.g., toll highways reserved for light-duty vehicles during predetermined time periods).

- Social commitment: Given their attributes, roads are the only transport mode that guarantees practically universal access for the movement of all users under more or less the same conditions, regardless of their economic or social status. The universal nature of this access, which dramatically affects the quality of the services offered, is what positions this transport mode as the primary and conceptual cornerstone of the economic and social development of an advanced country. A fact that also cannot be overlooked is that roads are used not only by personal vehicles and heavy equipment 
but also by mass transit vehicles that provide social service and economic accessibility, not to mention motorcycles and bicycles, amongst other vehicles.

- Economic contribution: This has always been and will always be one of the main advantages of highway networks and road transport. Roads bring an enormous positive impact to national economies via their contribution to the gross domestic product, government tax revenues and the creation of direct and indirect jobs.

- User-oriented roads: It is time to move on from the traditional concept that implies that the main "clients" of road infrastructures are always national or regional governments. The true clients are the citizens using these roads. With this in mind, Smart Roads are to be defined and re-defined on an ongoing basis taking into account the requirements and expectations of users.

- Technology and innovation: All the parameters above require a decided action from those public and private stakeholders active in the road-related scientific and technological arena. Innovation plays a key role on all levels, from the provision of more effective services to the development of new materials, road equipment, sustainable road maintenance methods and much more.

\section{Emotional Attributes}

It is also possible to identify a series of emotional attributes associated with the Smart Roads concept, insofar as they are linked closely to the structural aspects set out above, notably [4]:

- Reliability: Roads should provide assurance in terms of predictable travel time and, by extension, free traffic flow for as much of the day as possible. This calls consequently for the systematic use of advanced technologies for managing actual demand from one moment to the next.

- Safety: Roads should offer the highest safety levels that technical and technological progress to date allows, with a view to securing mobility with the lowest possible risk and minimising the probability of and mitigating the potential effects of accidents. These characteristics are to be taken into account for all users that may happen to use any given route.

- Security: Roads should be the most important part of an integrated system managing the risks associated to natural and man-made disasters, and in addition providing an adequate response and recovery time to all kinds of incidents.

- Comfort: Good driving conditions are an essential parameter for ensuring user satisfaction and must take into account adequate road visibility and signalling, even pavements and proper services alongside roads.

- Modernity: Insofar as no specific model has been defined for every possible typology for road travel, users do not generally classify roads as an "advanced" transport mode. Making a distinction between passenger and goods transport, between short, frequent trips and long-haul travel, etc., are key challenges to design a system perceived as modern and attractive by the public opinion.

- Freedom: Far from an association with fixed departure times or limited service offers that do not necessarily fulfil the needs of users, roads are synonymous with freedom. 


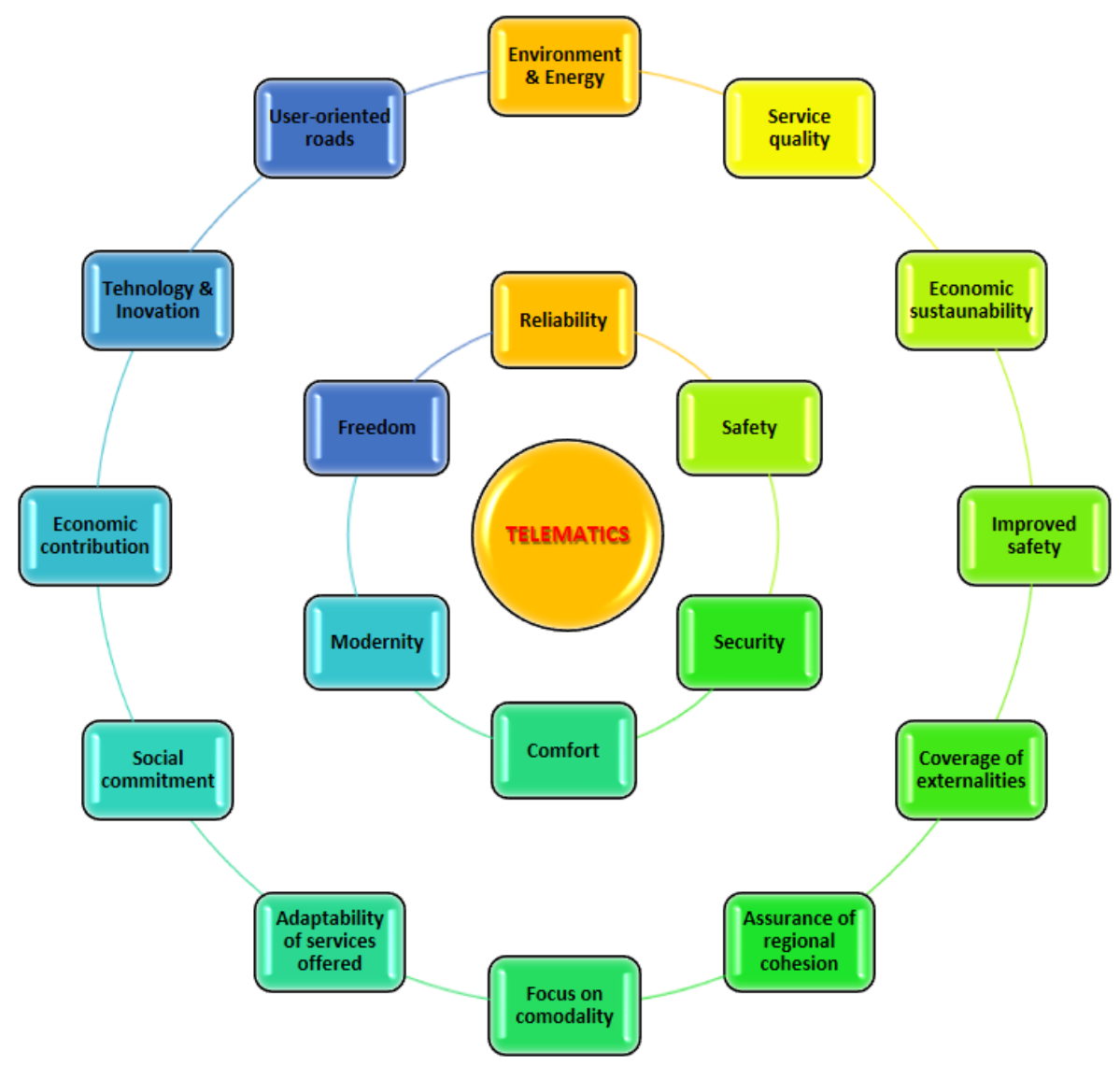

Figure 2. The key attributes of smart roads

Source: Author's own elaboration

\section{Conclusion}

Advanced telematics solutions are now implemented worldwide, with the goal harmonization of traffic flows, but also modernization of the entire transport system. Every year various projects, the purpose of which is based on the best possible modernization of highways, but also innovative telematics solutions that need to be integrated, the results are excellent driver awareness of road conditions.

Most of the experts believe that it is even possible to develop various solutions related to accidents, traffic congestion and emergency services. All these measures will change the way we currently consider highways and expressways by making them much safer and faster. Due to all these benefits, the demand for telematics has been significantly growing in almost every part of the world. In fact, many professional fleets and other such lines of businesses have already implemented this technology. Some automobile companies even use this technology in passenger cars. For people seeking more safety and greater efficiency, telematics is the perfect solution.

\section{References}

[1] Commission staff working document. EU Road Safety Policy Framework 2021-2030 - Next steps towards "Vision Zero", 1-3.

[2] Virtanen, A., Schirokoff, J., Luoma and R. Kulmala Impacts of an automatic emergency call system on accident consequences. Ministry of Transport and Communications. Finland 2006.

[3] HERE Mobility. Available at: https://mobility.here.com/learn/smart-transportation/smart-roadtechnology-paving-way-future [Accessed: October 09, 2020]

[4] Elena De La Peña, Smart Roads: A Vision, Spanish Road Association Chair, Spain 2015. 